Meeting report

\title{
Building bridges from 'omics' to cell biology Brian Cox
}

Address: The Hospital for Sick Children Research Institute, Program in Stem Cell and Developmental Biology, 101 College Street, Toronto, Ontario, Canada M5G 1L7. Email: b.cox@utoronto.ca

Published: 10 March 2009

Genome Biology 2009, 10:305 (doi:10.1 186/gb-2009-10-3-305)

The electronic version of this article is the complete one and can be

found online at http://genomebiology.com/2009//0/3/305

(C) 2009 BioMed Central Ltd

A report from the Keystone Symposium on Molecular and Cellular Biology, 'Omics Meets Cell Biology', Breckenridge, Colorado, 25-30 January, 2009.

A recent meeting in the Keystone series focused on the convergence of two fields, large-scale biology 'omics' (defined as anything with an 'omic' ending - genomics, proteomics, metabolomics, and so on) and cell biology, including yeast, mammalian tissue culture and animal models such as Drosophila, Caenorhabditis elegans and mouse. Ruedi Aebersold (Institute for Molecular Systems Biology, Zurich, Switzerland) explained the concept as two groups of researchers - the cell biologists who create islands of knowledge and the 'omicists' who try to connect these islands together.

Screens based on RNA interference (RNAi) are now widely used to uncover new members of signaling pathways and to assess gene function. An interesting extension of the image analysis typically used in cell-based RNAi screens was presented by Lucas Pelkmans (Institute for Molecular Systems Biology, Zurich, Switzerland) for screens designed to uncover cellular factors affecting viral infectivity. He showed that cell-based screens can be subject to a high degree of variability, potentially due to heterogeneity in the cell population. But by controlling for cell morphology and density, biases in viral infection that had previously confounded the comparison between normal and RNAi-treated cells, Pelkmans and colleagues were able to identify Dyrk kinases as affecting the infectivity of a wide range of viruses.

Other screens identified components of cell organelle formation. Laurence Pelletier (Samuel Lunenfeld Research Institute, Toronto, Canada) presented an RNAi analysis of 114 proteins previously identified as being enriched to the spindle pole in human cells. Forty of these gave statistically consistent phenotypes affecting the spindle pole. One of these, C140rf94, is part of a complex of eight proteins with homology to the Augmin complex from Drosophila, three other proteins of which were then also noted to be phenotypic in the screen. Marta Lipinski (Harvard Medical School, Boston, USA) presented an RNAi screen, using image analysis, for proteins involved in regulating autophagy (the digestion of a cell's own organelles) in mammalian cells. Lipinski concluded that this process was independent of the protein kinase mTOR (mammalian target of rapamycin) and appeared to be under the control of several signaling pathways, including that initiated by fibroblast growth factor, and is thus much more diverse than in yeast, where autophagy seems only to be dependent on TOR.

RNAi screens can also identify novel components in biological processes. Dan Durocher (Samuel Lunenfeld Research Institute, Toronto, Canada) presented a re-analysis of a published RNAi screen that has uncovered a new DNA damage response gene, $R N F 168$, involved in the RIDDLE syndrome (radiosensitivity, immunodeficiency, dysmorphic features and learning difficulties) in humans. Durocher showed that the RNF168 protein could ubiquitinate H2AX histones and acted downstream of the ubiquitin ligase RNF8 but upstream of MDC1 and the tumor suppressor BRCA1. Joan Brugge (Harvard Medical School, Boston, USA) presented an RNAi-based screen in MCF-10A cells (a nontumorigenic human breast epithelial cell line) to find factors that accelerate or inhibit collective cell migration, as assessed by time-lapse video. Many novel regulators of the cell adhesion molecule beta-catenin were uncovered. David Sabatini (Massachusetts Institute of Technology, Cambridge, USA) presented a pooled RNAi screen of the effects on cell survival of 1,100 metabolic genes in various human cancer cell lines. He found that cancer cells were more sensitive than normal cells to the loss of enzymes involved in metabolic detoxification processes, such as Nudix hydrolases (which hydrolyze a variety of nucleoside diphosphate derivatives) and enzymes involved in amino acid breakdown. This is possibly because of higher levels of toxic intermediates formed as a result of the higher metabolic rate of cancer cells compared with normal somatic cells. 
Although great insight into many biological processes has come from cell-based screens, complex developmental and behavioral processes require animal models. Jennifer Mummery Widmer (Institute for Molecular Biotechnology, Vienna, Austria) presented a comprehensive analysis of Notch signaling in Drosophila, using a large-scale RNAi transgenic approach involving 20,000 lines and 11,000 genes. Widmer identified more than 170 new effectors of Notch signaling, including proteins involved in vesicle trafficking, nuclear import, and the COP9 complex, a general regulator of protein degradation.

Ernst Hafen (Institute of Molecular Systems Biology, Zurich, Switzerland) described a study in Drosophila that bridged classical genetic screening and proteomic analysis. First, a genome-wide random mutagenic screen uncovered 47 genes involved in the regulation of head size. Hafen then directly assayed the protein-protein interactions of these 47 genes. Two genes giving a small head phenotype - Bunched and Madam - were shown to interact; overexpressing both genes together gave the complementary phenotype of a big head, which was not observed when either was overexpressed alone. Sabine Cordes (Samuel Lunenfeld Research Institute, Toronto, Canada) discused an ethylnitrosourea-based mutagenesis screen in mice that uncovered genes conferring differential sensitivity to serotonin, a neurotransmitter that has been associated with psychiatric disorders in humans. She described the discovery of three genes - one conferring hypersensitivity to serotonin, one conferring hyposensitivity, and one conferring age-onset hyposensitivity.

The field of metabolomics is particularly challenging as more than $10^{6}$ different chemicals are estimated to be synthesized in the body, excluding proteins, DNA and RNA. Edward Dennis (University of California School of Medicine, San Diego, USA) described the use of liquid chromatography/ mass spectrometry (MS) and custom-synthesized standards to quantify 220 different eicosanoid lipids, which include the prostaglandins and leukotrienes involved in inflammation. The platform has been applied to the investigation of Tolllike receptor signaling and Lyme disease. Peer Bork (EMBL, Heidelberg, Germany) presented an example of the integration of environmental and genomic data. Using metagenomic analysis, Bork analyzed a 1-cm deep block of soil at 2-mm intervals and found several regions with differing metabolic potential. Uwe Sauer (Institute for Molecular Systems Biology, Zurich, Switzerland) described the quantitative monitoring of 250 metabolic enzymes in yeast by MS under different growth conditions. Monitoring the flux of metabolites showed that, although individual metabolites were affected under different conditions, the net result of metabolite flux was similar, with the exception of switches of aerobic- versus fermentation-based metabolism.

Signaling networks have been difficult to elucidate, but the application of quantitative proteomics and phosphoproteomics is greatly advancing our understanding. Tony Pawson (Samuel Lunenfeld Research Institute, Toronto, Canada) presented an interesting study of bidirectional signaling involving the neural cell adhesion molecules Ephs and Ephrins. Using the technique of stable isotope labeling with amino acids in cell culture (SILAC) they differentially labeled cells expressing either protein, enabling measurement of changes in intracellular phosphopeptides in the presence or absence of signaling, as well as segregating Eph and Ephrin signal cascades. An RNAi screen was then used to functionally characterize the targets of Eph and Ephrin signaling.

Aebersold presented work on defining kinase substrate networks in yeast by phosphopeptide anlaysis in 120 different mutant kinase yeast lines. More than 900 differentially phosphorylated targets of the tested kinases were identified. In response to a question, Abersold said that there were differences between mutation and pharmacological inactivation of the same kinase, as the mutant strain is adapted to the loss whereas the pharmacological inhibition is instantaneous. Steven Gygi (Harvard Medical School, Boston, USA) described work aimed at identifying all targets of the cell-cycle-dependent kinase Cdk1. He found that Cdk1 target sites within compact protein domains were highly conserved across 32 different yeast species, whereas those in loops or disordered regions - $90 \%$ of all sites - were poorly conserved.

Oliver Hantschel (Austrian Academy of Sciences, Vienna, Austria) presented work using the immobilized tyrosinekinase inhibitors imatinib mesylate (Gleevec), bosutinib and dasatinib as affinity reagents to isolate kinases from cell lysates. Although bosutinib and dasatinib had higher affinity than Gleevec for the kinase Abl1, they had far greater offtarget kinase activity, binding more than 70 different kinases. Despite this, when cells with the Bcl-Abl translocation mutation were treated with the drugs, they had similar transcriptional profiles, suggesting that the off-target effects were not significant in the context of the cancer cell.

Garry Nolan (Stanford University, Stanford, USA) presented a stunning single-cell analysis of signaling changes in leukemias under different conditions, which had generated a content-rich dataset that facilitated the subdivision of the tumor into different tumor cell types based on the cells' signaling potentials. Nolan showed that this system could predict clinical outcomes through deep correlation mining. He also described the development of a hybrid flow sorter/ mass spectrometer that, can routinely measure the binding reactions of 14 different antibodies simultaneously, and has a capacity of up to 50 different antibodies.

Structural biology has greatly expanded in the past decade due to the establishment of several consortia. Cheryl Arrowsmith (Ontario Cancer Institute, Toronto, Canada) presented 
work focusing on the ubiquitination system. She and colleagues have systematically solved the structures of more than five E2 ligases to uncover their specificity for ubiquitinlike molecules such as Sumo. They carried out sequence clustering then solved representative cluster members. Next they performed enzymatic assays on the majority of them to classify their activities. Wolfgang Baumeister (Max Planck Institute of Biochemistry, Martinsreid, Germany) presented his work towards the goal of topological structural analysis of cell systems, using cryo-electron tomography to generate three-dimensional topological structures of macromolecules. He then identifies different macromolecular complexes in fixed cells, based on a library of topological structures.

A variety of useful resource websites were described at the meeting, including LipidMaps [http://www.lipidmaps.org], which provides data on lipid metabolic pathways and synthesis, and the Protein Atlas [http://www.proteinatlas. org], which aims to produce an antibody against every protein in the human genome. Currently there are data for antibodies against 6,000 genes; antibodies against the 20,000 known protein-coding genes is anticipated by 2010 . Other databases included a site containing genetic and proteomics data on many aspects of cell migration and motility [http://www.cellmigration.org], a new version of the String protein-interaction network [http://string.embl. de], the newest release of Cytoscape [http://www.cytoscape. org], an open-source Java application for viewing a range of data types as networks, and an update of Phosphositeplus [http://www.phosphosite.org], a database that integrates MS-observed phospho-peptides and other posttranslational modifications with multi-species alignments and protein structures.

At the end of the meeting there was a real impression that stamp collecting in omics-based research was over (finally). Research now involves function-based experimental design combined with omics scale and technologies. Omics technologies are no longer confined to the classical methods of genomics, proteomics and transcriptomics, but now include large-scale RNAi, overexpression, mutagenesis, and small-molecule analysis. Nor is the field largely confined to yeast, which in the past has been the staple of omics researchers, as the majority of projects presented at the meeting involved mammalian or insect cell culture and model organisms such as fly, worm and mouse. It is apparent that omics-based technologies have opened up cell biology to a new era of investigation.

\section{Acknowledgements}

The author thanks Jean Cox for reviewing the manuscript. 